\title{
Marshall SAHLINS, What Kinship Is-And Is Not
}

Filippo Zerilli

\section{(2) OpenEdition}

\section{Journals}

Édition électronique

URL : http://journals.openedition.org/ress/3168

DOI : $10.4000 /$ ress.3168

ISBN : $1663-4446$

ISSN : 1663-4446

Éditeur

Librairie Droz

Édition imprimée

Date de publication : 15 novembre 2015

Pagination : 282-285

ISSN : 0048-8046

Référence électronique

Filippo Zerilli, "Marshall SAHLINS, What Kinship Is -And Is Not », Revue européenne des sciences sociales [En ligne], 53-2 | 2015, mis en ligne le 15 novembre 2015, consulté le 22 septembre 2020 URL : http://journals.openedition.org/ress/3168 ; DOI : https://doi.org/10.4000/ress.3168

Ce document a été généré automatiquement le 22 septembre 2020

(C) Librairie Droz 


\title{
Marshall SAHLINS, What Kinship Is- And Is Not
}

\author{
Filippo Zerilli
}

\section{RÉFÉRENCE}

Marshall SAHLINS, 2013, What Kinship Is-And Is Not, Chicago, The University of Chicago Press, $110 \mathrm{p}$.

1 À en juger par son titre, What Kinship Is-And Is Not, on pourrait penser que le dernier livre de Marshall Sahlins, aussi ambitieux par le propos qu'il est petit par le volume, se propose d'expliquer au large public ce qu'est la parenté (et ce qu'elle n'est pas). Or, loin d'être un ouvrage de vulgarisation à propos d'un sujet classique et plutôt technique de l'anthropologie, écrit par l'un des anthropologues américains les plus « européens » de sa génération - l'auteur est né en 1930 -, il s'avère être la version finale d'un long essai scientifique déjà partiellement publié dans le Journal of the Royal Anthropological Institute (2011, 17-1, p. 2-19 et 17-2, p. 227-242), éminente revue de l'anthropologie sociale britannique la plus autorisée.

2 En dépit du remaniement qu'il a subi, le livre garde le style éclectique - et parfois elliptique - d'un essai s'adressant avant tout aux spécialistes. L'auteur le présente comme " a modest proposal for solving the 150-year-old anthropological problem of what kinship is » (p.ix). Pourtant, on n'y trouvera ni une analyse des grands débats sur la parenté ni, à proprement parler, un historique du domaine. L'auteur y esquisse une généalogie des études de parenté des travaux d'Émile Durkheim à ceux de Marilyn Strathern, en passant par les contributions de Lucien Lévy-Bruhl, Maurice Leenhardt ou Roger Bastide. Mais il le fait justement dans la mesure où ces auteurs - et quelques autres - forment le lignage dans lequel Sahlins lui-même inscrit son propos novateur. Ce dernier, annoncé dans un courte préface (p.ix-x), vise à montrer que la caractéristique principale de la parenté consisterait essentiellement dans la reconnaissance de la «mutualité d'existence » (mutuality of being), telle que les parents 
(kinfolk) « are persons who participate intrinsically in each other's existence; they are members of one another. [They] emotionally and symbolically live each other's lives and die each other's deaths » (p. ix). Qui ayant vécu l'expérience de la naissance ou de la mort d'un de ses proches pourrait le démentir?

Le livre compte seulement deux chapitres; le premier intitulé «What kinship is-Culture " et le second « What kinship is not-Biology». Dans le premier, beaucoup plus long que le second et organisé en plusieurs sections (p.1-61), l'auteur commence par situer sa démarche intellectuelle par rapport au constructivisme, qu'il considère comme l'orthodoxie en vigueur aujourd'hui dans les études d'anthropologie de la parenté. Tout en en réfutant de manière un peu hâtive ses dérives postmodernistes (p. 9-10), Sahlins observe que la prétention à considérer les relations de filiation, d'alliance et d'affinité comme des constructions sociales n'en est pas moins encore souvent hantée par un préjugé naturaliste qui consiste à reconduire de manière implicite la parenté aux faits naturels de la reproduction biologique et/ou à son articulation généalogique. Dans la section suivante, Sahlins revient sur l'analyse que David Schneider (A Critique of the Study of Kinship, 1984) avait consacré à un compte rendu célèbre de Durkheim paru dans le premier tome de L'Année sociologique (1898). S'il reproche au premier d'avoir conduit les études de parenté dans une impasse, il crédite cependant Durkheim d'avoir distingué la parenté de la généalogie, ce qui lui permet de réaffirmer la dimension sociale de la parenté et d'en montrer toute la spécificité. Pour Sahlins, la parenté n'est pas une simple invention occidentale (comme le voulait Schneider) : elle est bien un objet social réel qui s'exprime dans la «mutualité d'existence " (titre de la troisième section, p. 19-31); à savoir une modalité particulière de mise en relations des personnes entre elles qui est typique des humains (et qui les distingue des grandes singes, comme l'éclaire la section consacrée à une digression sur la nature humaine supportée par les acquis de la psychologie cognitive de Michael Tomasello et d'autres chercheurs, voir p. 48-54).

4 Marshall Sahlins affirme n'avoir pas la prétention de démontrer une thèse ni de la vérifier: il se contente d'illustrer son point de vue (p.2). Bien qu'il fasse parfois référence à des travaux anciens ou même classiques (à l'exemple des Researches into the Early History of Mankind d'Edward Burnett Tylor [1865], dont il évoque la notion de couvade, ou de l'Éthique à Nicomacque d'Aristote), il se sert surtout d'une quantité impressionnante de sources ethnographiques récentes, privilégiant les données concernant des populations qu'en France on appelait naguère "ethnologiques". Suivant un procédé de "comparaison incontrôlé ", qu'il qualifie ironiquement de "style frazerien", Sahlins amasse plusieurs dizaines d'exemples pour montrer comment les idéologies et les pratiques de parenté se fondent sur la participation intersubjective (intersubjective participation) des substances (soient-elles biologiques, matérielles ou mentales) entre " parents ». Pour les Korowai de Nouvelle Guinée (selon Rupert Stasch) devenir parents signifie se rendre visite réciproquement, coopérer, offrir de la nourriture. Chez les Inuit du Groenland (étudiés par Mark Nuttall) on peut être parents lorsqu'on est né le même jour, ou si l'on se donne le même nom. Lorsqu'un enfant prend le nom d'un parent disparu - par exemple un grand-père maternel - il s'adresse à sa mère biologique comme à sa "fille", au mari de celle-ci comme à son "beau-fils" et à sa grand-mère comme à sa "femme". Certains populations de Malaisie (nous apprend Janet Carsten) acquièrent le même "sang " en vivant dans la même maison et en se nourrissant de le même terre. Pour les Ilingots des Philippines (selon Renato Rosaldo), ceux qui partagent une histoire de migration partagent le 
même corps. Chez les Achuar d'Amazonie (étudiés par Anne-Christine Taylor) la personne et son corps sont constitués par un ensemble d'éléments (les noms, le sang, les os, la décoration, la capacité de parler ou d'agir, etc.) acquis ou hérités comme membres d'une collectivité. Enfin, la notion de parenté s'élargit aux relations que certaines populations établissent avec d'autres espèces animales (Amazonie, Sibérie) ou végétales (Nouvelle Calédonie, et encore Amazonie).

Cette revue de la littérature ethnographique représente un tour de force fructueux parce que Sahlins, loin de se limiter à ramasser des données, retravaille les suggestions théoriques provenant des différentes modalités d'élaboration des relations de "mutualité d'existence», y compris celles développées à partir de la notion de " dividual person" rendue célèbre par les études de Marilyn Strathern en Mélanésie (1988) et introduite pour la première fois par McKim Marriot en 1976. Sans leur épargner quelques critiques ponctuelles, Sahlins reconnaît volontiers ses dettes aussi bien à ses prédécesseurs qu'à ces contemporains. Parmi ces derniers, il rend notamment hommage à Eduardo Viveiros de Castro, représentant influent du tournant ontologique en anthropologie. Ainsi, c'est à la «mystérieuse efficacité de la relationalité » (selon l'expression de l'anthropologue brésilien) qu'est consacrée la dernière section du premier chapitre (p. 58-61). Sahlins reprend à nouveaux frais l'intuition de Viveiros de Castro selon lequel la parenté appartiendrait, avec l'échange de dons et la magie, à un seul et même ordre animiste: " $a$ world where yams are our lineage brothers and roam unseen at night, or where jaguars strip away their animal clothes and reveal themselves as our cannibal brothers-in-law» (Viveiros de Castro, cité par Sahlins, $\mathrm{p}$. 60). Ce faisant, le " risk of dragging the discussion of kinship into dark philosophical waters ", que Sahlins avait déclaré vouloir éviter (p. 32), se fait tout de même assez pressant.

Dans le second chapitre (p. 62-89), Sahlins prolonge son propos en insistant notamment sur la dimension non-biologique, ou pré-biologique, de la parenté. Avec une verve polémique qui n'étonnera pas les lecteurs de son ouvrage The Use and Abuse of Biology (1976, traduit en 1980 : Critique de la sociobiologie, Gallimard), Sahlins entend notamment démanteler l'idée que les catégories de parenté seraient des représentations ou des métaphores de la naissance et de la procréation. Selon lui, c'est plutôt l'inverse qui est vrai: "human birth is a semiotic function of a kinship order, rather than kinship a biological sequitur of birth» (p. 87). Ainsi, avec d'autres exemples ethnographiques à l'appui, il propose un renversement du sens commun scientifique produit par les sociobiologistes et les psychologues évolutionnistes, mais partagé aussi par les anthropologues travaillant dans des perspectives "egocentriques» ou "extensionnistes» (p. 80). L'objection n'est pas sans conséquences sur le lexique de parenté et les approches terminologiques (que faire de la notion de "parenté fictive ", par exemple ?) et soulève quelques doutes sur l'utilité des tentatives de traduire l'expérience du devenir parents dans les langages formels inspirés du structuralisme (à quoi sert l'algèbre de la parenté ?).

7 What Kinship Is-And Is Not est un livre complexe, subtil et important. Depuis sa publication en 2013, il n'a pas cessé d'interpeller les spécialistes (voir notamment le book symposium organisé par HAU: Journal of Ethnographic Theory, 2013, 3-2, p. 245-316). Finalement, il peut être lu comme une réponse - tardive mais brillante - au questionnement radical lancé par Schneider il y a plus que quarante ans («What is Kinship All About? ", 1972) et à la progressive marginalisation du domaine qui suivit l'analyse critique corrosive que ce dernier en fit en 1984. Si Schneider y fait figure de 
grand absent, le mérite principal du livre de Sahlins consiste à avoir produit une remarquable synthèse critique des new kinship studies, lesquelles sont restées quelque peu souterraines pendant les vingt dernières années, en dépit de leur importance et de leur vitalité. C'est grâce à la diversité et à la qualité de ce riche corpus ethnographique que Sahlins a pu mener à bien son entreprise. Ainsi, ce maître incontesté de l'anthropologie, sans être un spécialiste de la parenté - et peut-être justement grâce à cela - a-t-il réussi à remettre ces études au centre du débat anthropologique contemporain. Et ne serait-ce que pour cela, il faut l'en féliciter. Cependant, pareille opération présente au moins deux limites. D'abord, elle tend à reproduire une image un peu vieillotte de la discipline entendue comme l'étude des cultures exotiques, façon " among the So-and-So ", un genre que - paradoxalement - Sahlins avait lui-même contribué à mettre en question. Ensuite, il paraît légitime de se demander si la notion de «mutualité d'existence », loin d'être réservée à l'analyse de la parenté, ne pourrait être aussi utile à l'exploration d'autres domaines de la vie sociale - tels par exemple les idées et les pratiques religieuses ou celles concernant le fonctionnement de l'État capitaliste à l'ère du néo-libéralisme -, et ce, dans la mesure où les « kin persons are not the only kind who are multiple, divisible, and relationally constructed » (p. 27). Si l'on poussait cette remarque un peu plus loin, l'esprit de notre ancêtre Schneider pourrait se réveiller, quoique inévitablement sous des formes différentes.

\section{AUTEURS}

\section{FILIPPO ZERILLI}

Université de Cagliari - DISSI 